\title{
La phénylcétonurie, du golfe du Morbihan à l'estuaire du Saint-Laurent... Un marqueur de démographie historique inattendu !
}

La phénylcétonurie (PCU) classique résulte du déficit d'une enzyme d'expression spécifiquement hépatique, la phénylalanine hydroxylase $(\mathrm{PAH}$ [1]). En l'absence de traitement, ce déficit est responsable d'une arıération mentale sévère, et chaque année naissent en France 60 à 80 enfants atteints de cette maladie autosomique récessive. Le dépistage systématique des hyperphénylalaninémies chez tous les nouveau-nés a permis la prévention du retard mental dans les pays développés par la mise en œuvre d'un régime contrôlé en protides. En outre, il a conduit à identifier de nombreuses formes variantes de la maladie.

Depuis son clonage par le groupe de S. L. C. Woo en 1986, le gène de $\mathrm{PAH}$ a été particulièrement étudié. Il est localisé sur le chromosome $12 \mathrm{q}$ et couvre un vaste domaine d'ADN de l'ordre de $90 \mathrm{~kb}$ [2]. Il est particulièrement riche en polymorphismes de restriction définissant un grand nombre d'haplotypes et donc d'allèles à ce locus [3].

Quant à la pathologie moléculaire du gène de $\mathrm{PAH}$, elle tend à rejoindre lentement celle du gène de $\beta$-globine, tant sont nombreuses les mutations décrites à ce jour (plus de 60). La combinatoire de toutes ces mutations est très probablement à l'origine du large spectre de tolérances protéiques observées chez les enfants [4].

En conjuguant l'étude des haplotypes à celle des génotypes, plusieurs équipes ont pu formuler des hypothèses séduisantes quant à l'origine géographique, historique ou ethnique des mutations. En effet, si une même mutation peut survenir fortuitement dans le même gène en deux points distincts du globe, il est très peu probable qu'elle survienne sur deux gènes d'haplotypes identiques. Dès lors, deux individus porteurs de la même mutation et de gènes de $\mathrm{PAH}$ d'haplotypes identiques sont vraisemblablement des cousins éloignés! Un gène très polymorphe, dont les mutations conduisent à une maladie facilement détectable, devient donc un très bon " traceur " de démographie historique.

Le premier traceur identifié en matière de phénylcétonurie fut la mutation R408W. Cette mutation est étroitement associée à l'haplotype 2 en Europe du Nord [3], en Europe de l'Est et particulièrement en Pologne, où sa fréquence avoisine 60 à $80 \%$ des gènes mutants [5]. Un gradient décroissant d'est en ouest a été constaté et cette mutation n'est plus responsable que de $30 \%$ des allèles mutants en Allemagne, de 10 à $15 \%$ au Danemark et en Angleterre, et de $7 \%$ en France [4]. Cette observation suggère que la mutation $\mathrm{R} 408 \mathrm{~W}$ est apparue en Europe de l'Est avant de se propager vers l'ouest selon un gradient opposé à celui de la mutation $\Delta$ F508 de la mucoviscidose.

Utilisant une large délétion du gène de PAH comme marqueur dans la population juive yéménite [6], le groupe de Yossi Shiloh, à Tel-Aviv, a pu de même identifier très exactement l'ancêtre fondateur de cette délétion à San'a, capitale historique du Yémen, avant le XVII e siècle.

Dans un même ordre d'idées, nous avons formulé une hypothèse hardie relative à l'origine d'une mutation au codon 280 (E280K). Nous avons en effet observé cette mutation en association étroite avec un haplotype de restriction très rare, sur quelques chromosomes originaires d'Afrique du Nord et, de manière surprenante, sur deux chromosomes... bourguignons depuis plus de 350 ans ! Cette observation avait donné lieu à un article dans méde- cine/sciences intitulé "Base moléculaire de la phénylcétonurie en France: de l'invasion celte à la bataille de Poitiers " [7]. Elle avait, hélas, occasionné aussi en son temps quelques controverses scientifiques mais également, dans un journal satirique ("Français sang pour sang ", le Canard enchaîne), quelques lignes amères relatives au droit de regard du généticien sur l'histoire des hommes et de leurs migrations [8].

A l'inverse, utilisant cette même confrontation des haplotypes avec les mutations, la découverte de mutations identiques sur des chromosomes porteurs d'haplotypes différents nous a conduits à l'hypothèse de la récurrence de certaines mutations [9]. Ces mutations récurrentes surviennent le plus souvent dans un dinucléotide $\mathrm{CpG}$ dont on sait qu'il constitue un point chaud de mutations.

Plus récemment, le groupe de Charles Scriver et Rima Rozen a identifié une mutation ponctuelle au codon d'initiation de la traduction du gène de $\mathrm{PAH}$ chez des Canadiens français (mutation M1V [10]). Cette mutation, responsable d'un déficit profond en $\mathrm{PAH}$, est étroitement liée à l'haplotype 2. Nous venons de rapporter les résultats d'un travail visant à identifier l'origine des fondateurs de la mutation M1V au Québec, par la recherche de cette mutation chez un grand nombre de patients originaires de France [11]. Ce dépistage, rendu possible par l'abolition d'un site de restriction NlaIII par la mutation M1V, a été effectué sur les cartes de Guthrie d'enfants suivis par de nombreux collègues généticiens en France [8]. Sur les 152 gènes testés, quatre seulement portaient la mutation M1V. Fait surprenant, ces quatre allèles ont été découverts dans des familles vivant en Bretagne, à proximité du golfe du Morbihan, où elles sont ins- 
tallées depuis plus de quatre générations. En revanche, cette mutation n'a été trouvée nulle part ailleurs, en particulier pas dans les régions voisines, pourtant connues pour avoir largement contribué aux migrations vers le Québec (Anjou, Poitou, Maine, 40 chromosomes testés). Elle est également absente de 71 chromosomes mutants originaires du reste du territoire français. Le regroupement de ces trois familles dans un diamètre de $50 \mathrm{~km}$ suggère qu'on a affaire à un seul et même chromosome ancestral. De plus, il est remarquable que les allèles observés en France sont tous d'haplotype 2, ce qui rend hautement vraisemblable une communauté d'origine des allèles bretons et canadiens.

Enthousiasmés par cette observation qui permettait de documenter les liens généalogiques qui nous unissent, nos amis canadiens des universités de McGill et de Chicoutimi ont effectué, de leur côté, des reconstructions généalogiques, utilisant des logiciels développés par la SOREP, qui visent à identifier les origines géographiques des Canadiens français contemporains [12]. De façon surprenante, ces reconstructions ont permis d'identifier pas moins de 53 fondateurs potentiels communs pour les sept patients porteurs de la mutation M1V au Québec moderne, tous originaires de France mais aucun de Bretagne ! En revanche, 19 d'entre eux $(35,8 \%)$ étaient originaires de Mortagne, dans le Perche, qu'ils avaient quitté pour le Canada au XVII siècle. De fait, le Perche a largement contribué à la fondation du Canada français puisqu'on y a dénombré 202 émigrants $(7,4 \%)$ avant 1670, puis 15 de 1670 à $1760[13,14]$.

Pour rendre compte des deux origines géographiques distinctes de la mutation M1V en France, l'une dans le Perche et l'autre dans le Finistère sud, l'hypothèse de migration de populations du Perche vers la Bretagne (ou inversement) a été avancée, mais elle est peu vraisemblable compte tenu de l'éloignement de ces régions $(300 \mathrm{~km})$ et de la sédentarité de nos ancêtres dans la France du XVIIe siècle. Bien entendu, les reconstructions généalogiques sont sujettes à de nombreuses inexactitudes comme, par exemple, les fausses paternités et les adoptions. Toutefois, ces contemporain - ne sont pas nécessairement incompatibles. Entre 1608 et 1699, l'immigration observée au Québec a été évaluée à 14400 individus par Marcel Trudel ainsi que par Charbonneau $[13,15]$. De ces fondateurs du noyau historique du Québec, plus de $70 \%$ sont retournés en France [13, 15], essentiellement des engagés en vertu de la charte octroyée en 1627 par Colbert, des "filles à marier " et des militaires... Ainsi, le bilan net de l'immigration pionnière dans le Québec du XVIIe siècle représente à peine 4990 individus d'après ces mêmes auteurs. Le lieu de retour des pionniers déçus est beaucoup moins bien connu que l'origine de leurs ancêtres. Ainsi, n'est-il pas invraisemblable que le regroupement contemporain des allèles M1V dans le golfe du Morbihan soit lié au retour de ces Québéquois sur les côtes françaises, en Bretagne, à $300 \mathrm{~km}$ de leur point de départ historique.

Il n'était probablement pas inutile que médecine/sciences, trait d'union scientifique entre la France et le Canada, se fasse l'écho de ce type de collaborations franco-canadiennes, d'autant que d'autres affections particulières aux Canadiens Français - comme la maladie de Tay Sachs ou la tyrosinémie héréditaire - pourraient faire à leur tour l'objet d'études de démographie historique qui pourraient mener les généticiens sur les traces des navigateurs : du golfe du Morbihan à l'estuaire du Saint-Laurent

Stanislas Lyonnet, Dominique Melle, Françoise Rey, Jean Rcy, Arnold Munnich

Unité de recherches sur les handicaps génétiques de l'enfant, Inserm U. 12 et département de pédiatrie, hôpital des EnfantsMalades, 149, rue de Sevres, 75743 Paris Cedex 15, France.

Charles R. Scriver

Children's Hospital Research Institute and McGill University, Montréal, Canada.

\section{TIRÉS A PART}

S. Lyonnet.

\section{RÉFÉRENCES}

1. Scriver CR, Kaufman S, Woo SLC. The hyperphenylalaninemias. In : Scriver CR, Beaudet AL, Sly WS, Valle D, eds. The Metabolic Basis of Inherited Diseases, 6th ed. New York : McGraw-Hill, 1989 : 495-546.

2. Dilclla AG, Kwok SCM, Ledley FD, Marvit J, Woo SLC. Molccular structure and polymorphic map of the human phenylalaninc hydroxylase gene. Biochemistry 1986; 25 : 743-9.

3. Chakraborty R, Lidsky AS, Daiger SP, et al. Polymorphic DNA haplotypes at the human phenylalanine hydroxylase locus and their relationship with phenylketonuria. Hum Genet 1987 ; 76 : 40-6.

4. Berthclon M, Caillaud C, Rey F, et al. Spcctrum of phenylketonuria mutations in Western Europc and North Africa, and their relation to polymorphic DNA haplotypes at the phenylalanine hydroxylase locus. Hum Genet 1991 ; 86 : 355-8.

5. Jaruzclska J, Henriksen KJ, Güttler F, et al. The codon 408 mutation associated with haplotypc 2 is predominant in Polish families with phenylketonuria. Hum Genet 1991; 86 : 247-50.

6. Avigdad S, Cohen BE, Bauer S, et al. A single origin of phenylketonuria in Yemenite Jews. Nature 1990 ; 344 : 168-70.

7. Lyonnct S, Rey F, Caillaud C, Abadie V, Munnich A, Rey J. Bases moléculaires de la phénylcćtonuric en France : de l'invasion celtc à la bataillc de Poitiers. médecine/sciences 1988 ; 4: 544-52.

8. Rey J, Lyonnet S, Rey F, Caillaud C, Munnich A. RFLP haplotype analysis and PCR amplification of dried blood spots in phenylketonuria : ethnics and ethics. In : Knoppers BM, Laberge CM, eds. Genetic Screening from Newborns to DNA Typing. Amsterdam : Elscvier Science Publ. 1990 : 167-77.

9. Abadic V, Lyonnct S, Maurin N, et al $\mathrm{CpG}$ dinuclcotides are mutation hot spots in phenylketonuria. Genomics 1989 ; 5 : 936-9.

10. John SWM, Rozen R, Laframboisc R, Labcrge C, Scriver CR. Novel PKU mutation on haplotype 2 in French-Canadians. $A m$ J Hum Genet 1989 ; 45 : 905-9.

11. Lyonnet S, Melle D, de Braekelecr M. et al. Time and space clusters of the French Canadians M1V phenylkctonuria mutation in Francc. Am J Hum Genet 1992 ; 51 : 191-6.

12. De Brackeleer M. A package for genetic analysis of pedigrees. SOREP, université de Québec à Chicoutimi. Thèse, 1991.

13. Charbonneau H, Guillemette A, Legaré J, Desjardins B, Laudry Y, Nault S. De l'ancicnnc à la nouvelle France : un mouvcment migratoire restrcint. In : Naissance d'unc population. Les Français établis au Canada au XVIII siècle. Travaux et Documents, vol. 118. Cahiers de l'INED, 1987.

14. Bolcda M. Les migrations au Canada sous le régime français (1608-1760). Cahiers Québéquois de Dénographie 1984 ; 13 : 23-39.

15. Trudcl M. La scigncuric des CentAssociés. In : la Société, histoire de la NouvelleFrance, vol. 3. Montréal : Fides, 1983. 